\title{
3D particle track reconstruction in a single layer cadmium-telluride hybrid active pixel detector
}

\author{
Mykhaylo Filipenko ${ }^{\mathrm{a}}$, Thomas Gleixner ${ }^{\mathrm{b}}$, Gisela Anton, Thilo Michel \\ University of Erlangen-Nuremberg, Erlangen Centre for Astroparticle Physics, Erwin-Rommel-Straße 1, 91058 Erlangen, Germany
}

Received: 25 March 2014 / Accepted: 30 July 2014 / Published online: 16 August 2014

(C) The Author(s) 2014. This article is published with open access at Springerlink.com

\begin{abstract}
In the past decades the search for neutrinoless double beta decay has driven many developments in all kind of detector technology. A new branch in this field are highly-pixelated semiconductor detectors-such as the CdTe-Timepix detectors. It comprises a cadmium-telluride sensor of $14 \mathrm{~mm} \times 14 \mathrm{~mm} \times 1 \mathrm{~mm}$ size with an ASIC which has $256 \times 256$ pixel of $55 \mu \mathrm{m}$ pixel pitch and can be used to obtain either spectroscopic or timing information in every pixel. In regular operation it can provide a two dimensional projection of particle trajectories; however, three dimensional trajectories are desirable for neutrinoless double beta decay search and other applications. In this paper we present a method to obtain such trajectories. The method was developed and tested with simulations that assume some minor modifications to the Timepix ASIC. Also, we were able to test the method experimentally and in the best case achieved a position resolution of about $63 \mu \mathrm{m}$ for electrons with an energy of $4.4 \mathrm{GeV}$.
\end{abstract}

\section{Introduction}

The main motivation behind the methods and experiments presented in this publication is to demonstrate the possibility of three dimensional (3D) particle trajectory reconstruction within the sensor of a hybrid active pixel detector for a neutrinoless double beta experiment. 3D tracking could help to achieve high sensitivities due to background rejection by particle identification. The neutrinoless double beta decay is a hypothetical, lepton-number violating decay where two neutrons in a nucleus are transformed into two protons and two electrons without anti-neutrino emission, e.g. ${ }^{1}$

\footnotetext{
1 The decay of ${ }^{116} \mathrm{Cd}$ could in principle be observed with a cadmiumtelluride semiconductor detectors, wherefore this particular isotope is chosen as an example here.

a e-mail: mykhaylo.filipenko@physik.uni-erlangen.de

be-mail: thomas.gleixner@physik.uni-erlangen.de
}

${ }^{116} \mathrm{Cd} \rightarrow{ }^{116} \mathrm{Sn}+2 e^{-}$.

This lepton-number violating decay is forbidden in the standard model of particle physics. Its observation would prove that neutrinos are Majorana fermions via the SchechterValle theorem [1]. This would require modifications in the standard model. The standard model distinguishes between neutrinos and anti-neutrinos and treats them as massless particles. The observation of neutrino oscillations already revealed that neutrinos have non-zero rest mass. If neutrinos were Majorana fermions, the small but non-zero neutrino mass (compared to the masses of the charged leptons) might be explainable with the help of sea-saw mechanisms. Therefore, besides the direct evidence for lepton-number violation, the observation of neutrinoless double beta decay would have an immense impact on our understanding of particle physics [2].

Although the search for the neutrinoless double beta decay is the main reason for our investigations, semiconductor voxel detectors could have other interesting applications as well. When using a thin sensor coupled to a pixelated ASIC, the read-out volume of single pixel is a box with pixel area as ground area and the sensor thickness as height, in our case $55 \times 55 \times 1000 \mu^{3}$. In order to resolve $3 \mathrm{D}$ particle tracks inside the sensor volume it is necessary to obtain position resolution along the height of the sensor. We name a detector that is able to perform this task a voxel detector.

As high resolution voxel detectors, semiconductor detectors might be used for efficient high energy single photon Compton-imaging. The direction of origin of an impinging $\mathrm{X}$-ray photo could be determined by reconstructing the track of the Compton scattered electron. Further, it could allow the usage for low activity tracers in SPECT imaging as the collimator, which absorbs most of the flux, could be avoided. Additionally, Compton-imaging could be used for security application; for instance, if a method for fast and precise detection of nuclear contamination is needed $[3,4]$. 


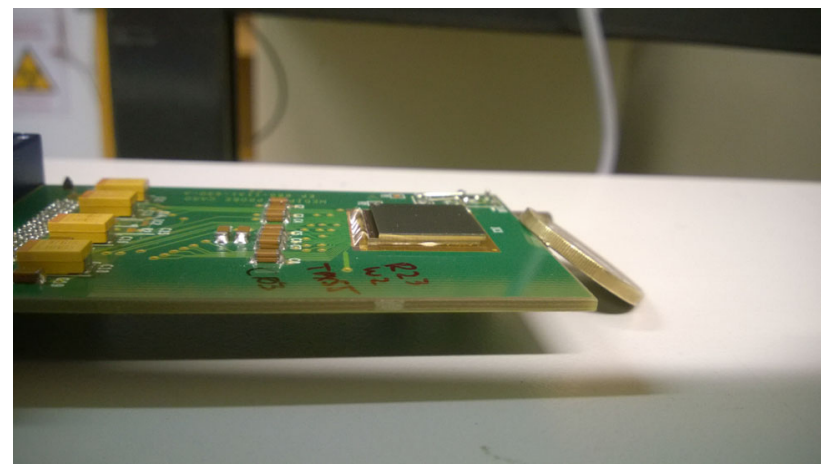

Fig. 1 A Timepix detector with a $1 \mathrm{~mm}$ thick cadmium-telluride sensor

3D imaging of particle tracks could also allow to perform a variety of other important low-background experiments like the observation of the double electron capture. Summing up, the development of voxel semiconductor detectors would enhance the possibilities of detection in various fields.

In this publication we present a method for the reconstruction of such 3D trajectories through the sensor layer. It was developed and optimized with simulations. Also, the method could successfully be applied to experimental data obtained with a Timepix detector [5] and used for the reconstruction of electron tracks through the sensor layer.

The paper is structured as follows: In the second section the Timepix detector is explained as our simulations and experiments are based on this technology. In the third section the reconstruction method is described and in the fourth section the experimental results are presented. Section 5 gives an outlook on the current detector development which could lead to a fully operable voxel detector with about $50 \mu \mathrm{m}$ voxel size.

\section{The Timepix detector}

The Timepix detector (s. Fig. 1) is a pixelated hybrid semiconductor detector which was originally developed for Xray imaging applications [5]. Hybrid in this context means that the sensitive sensor layer and the ASIC are fabricated separately and afterwards connected together via bumpbonds. This has the advantage that different semiconductor materials can be used for the sensor layer. For X-ray imaging it is desirable to have a sensor material with a high atomic number in order to achieve high absorption efficiency. Hence, besides silicon cadmium-telluride was established as a sensor material for Timepix devices. Despite the difficulty of obtaining large high quality cadmium-telluride crystals the advantage of room-temperature operation and an atomic number of 48 and 52, respectively, makes this material preferable (to germanium) for X-ray imaging applications.

A scheme of the Timepix structure and functionality is shown in Fig. 2. The Timepix used in this study consists

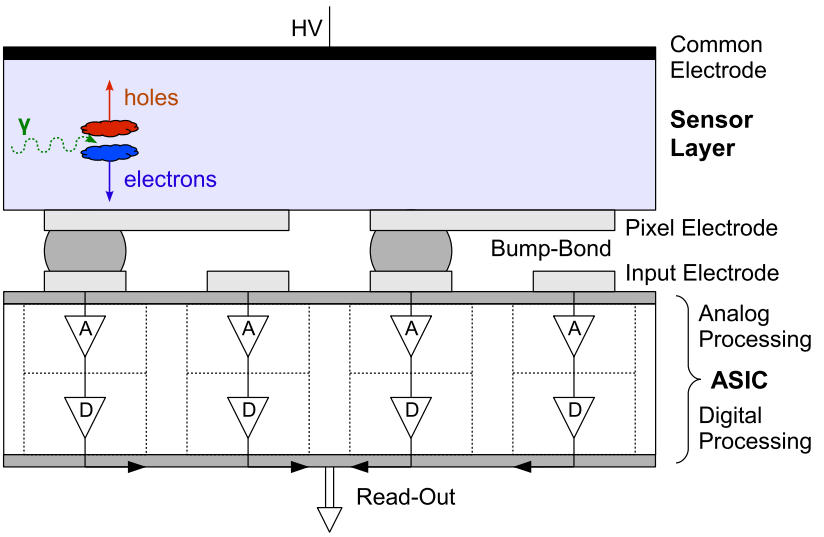

Fig. 2 Schematic cut through a Timepix-detector with a CdTe sensor layer with and block diagram that illustrates the signal processing in the $A$ analogue and $D$ digital parts of the ASIC. A high-voltage is applied to the sensor volume. The common electrode is at negative $\mathrm{HV}$, the pixel electrodes at approximate ground potential. Electrons and holes are generated by ionizing radiation and drifted by the electric field. During the drift the charge carriers induce mirror charge currents at the pixel electrodes which are integrated and converted to a voltage pulse by the analog electronics in the ASIC

of a $1 \mathrm{~mm}$ thick cadmium-telluride sensor with metal electrode pads (ohmic contacts) as pixel electrodes at the bottom and a common electrode for the high-voltage connection at the top. The sensitive sensor layer is connected pixel-bypixel to the input pixel electrodes of the ASIC by indium-tin bump-bonds. The pitch of the input pixel electrodes is 55 $\mu \mathrm{m}$. There are $256 \times 256$ pixels on the ASIC which corresponds to an active area of $1.4 \times 1.4 \mathrm{~cm}^{2}$. In our detector only every second pixel in each row and column is bumpbonded wherefore the effective pixel size is $110 \mu \mathrm{m}$. This has the advantage of better energy resolution at the cost of position resolution. The ASIC is mounted on a printed circuit board and connected to the computer by a USB-FitPix readout [6] for data transfer to the data acquisition computer which can provide up to 60 frames per second. The output data is provided in frames. Each frame is a $128 \times 128$ matrix of values obtained for every pixel during the frame-time. The frame-time can be preset manually to a fixed time or the frame-start/frame-stop can be controlled externally by a trigger.

The sensor layer can be biased with a voltage up to 800 $\mathrm{V}$. Free electrons and holes produced by ionizing radiation are separated and afterwards drifted by an electrical field in opposite directions. While approaching the pixel electrode the charge cloud induces mirror charges in the pixel electrodes. In each pixel the induced charge is collected and converted into a triangularly shaped pulse by a Krummernacher type preamplifier with an approximate peaking time of about $100 \mathrm{~ns}$. The length of the pulse is about several microseconds which is mainly determined by the falling edge of the pulse. 


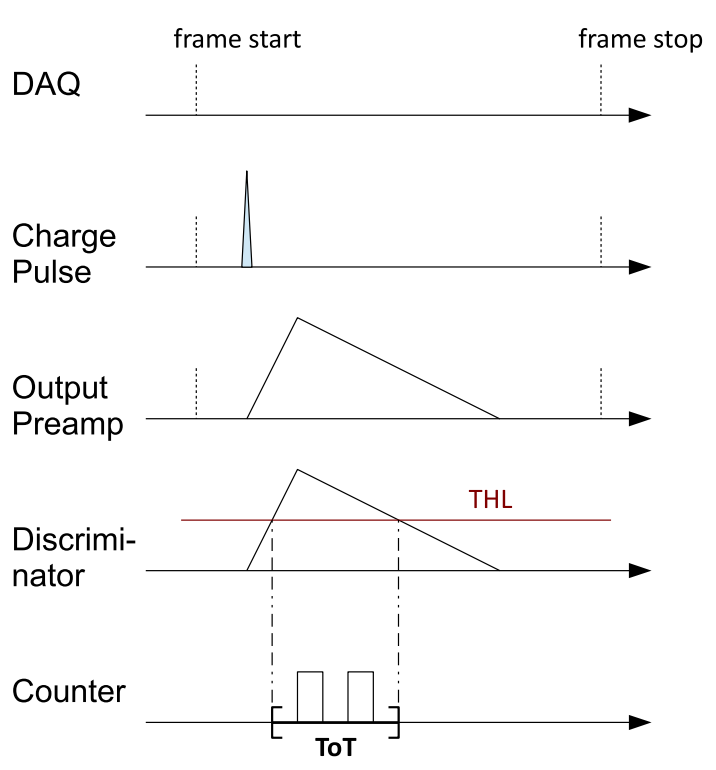

Fig. 3 Operation of the Timepix in the "time-over-threshold" (ToT) mode. After the charge pulse is amplified and converted to a voltage pulse, it is discriminated against a threshold level (THL). The timeover-threshold is the number of clock counts in the time interval of the pulse above the THL

The gain and the slope of the falling edge of the preamplifier can be set externally by a digital-to-analog converter in the matrix periphery. The pulse in each pixel is discriminated against a global threshold.

The electric post-processing of the signal in every pixel is digital and can be carried out in three different ways. We used the detector only in two modes - the "time-over-threshold" (ToT) mode and the "time-of-arrival" (ToA) mode. Both modes are illustrated in Figs. 3 and 4, respectively.

In the ToT mode a digital register starts counting clock cycles when the pulse rises over the threshold. The register stops once the pulse falls below the threshold and the number of counts in-between is the time-over-threshold. The frequency of clock cycles can be chosen between 10 and 100 MHz. The time-over-threshold is related to the height of the pulse which is a measure for the energy deposited in a pixel. Thus, the time-over-threshold can provide calorimetric information about a detected event. The deposited energy and the ToT are related in a non-linear way. The energy resolution achievable in this mode is $8.0 \%$ (FWHM) at $59.56 \mathrm{keV}$ and $5.4 \%$ (FHWM) at $122.06 \mathrm{keV}$. Details on the calibration and the energy resolution can be found in [7].

In the ToA mode the register starts counting on the rising edge of the pulse and stops only at the end of the frame. As every frame gets a global time stamp, the ToA provides a timing information for every event. It can be as good as 10 ns for every pixel if a $100 \mathrm{MHz}$ clock is used. The maximum counter value is 11280 which corresponds to a frame-time of $112.8 \mu$ s at a $100 \mathrm{MHz}$ clock frequency.

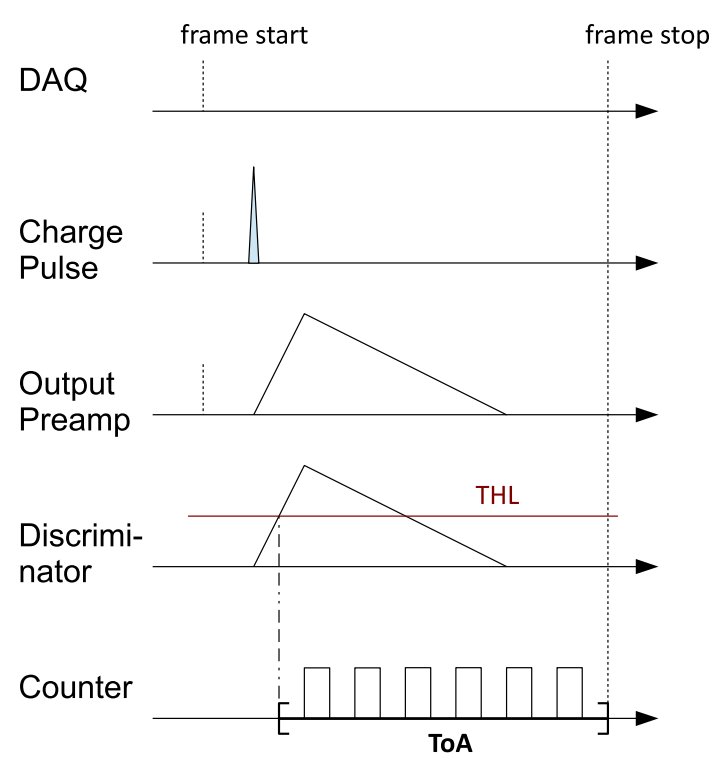

Fig. 4 Operation of the Timepix in the "time-of-arrival" (ToA) mode. After the charge pulse is amplified and converted to a voltage pulse, it is discriminated against a threshold level (THL). The time-of-arrival is the number of clock counts between the first time the voltage pulse rises above the THL and the end of the frame

\section{Reconstruction method}

In order to reconstruct the three-dimensional structure of particle trajectories through the sensor material, it is necessary to determine the depth of interaction $z_{n}^{0}$ in the sensor for every triggered pixel $n$ (Fig. 5). Without loss of generality, we assume that the sensor is pixelated in the xy-plane and the depth of interaction is to be calculated along the $\mathrm{z}$-axis. The sensor anode is at $z=0$ and the cathode is at $z=d ; d$ being the sensor thickness.

\subsection{Principle of reconstruction}

The reconstruction is based on the charge carrier propagation mechanism in the sensor layer. Charge carriers are drifted in z-direction due to an electric field that can be approximated in a CdTe sensor layer by

$\left|E_{z}(z, U)\right|=U\left(f_{2}+f_{1} z+f_{3} \exp \left(-f_{4} U z\right)\right)$

which is an empirical fit to experimental data [8]. The parameters $f_{1}$ to $f_{4}$ are $f_{1}=(5.80 \pm 0.09) \cdot 10^{5} \mathrm{~m}^{-2}$, $f_{2}=(228 \pm 7.5) \mathrm{m}^{-1}, f_{3}=(540 \pm 144) \mathrm{m}^{-1}$ and $f_{4}=(479 \pm 216)(\mathrm{Vm})^{-1}$. U is the voltage difference applied to the sensor volume. The drift velocity for electrons can be calculated as $v_{z}(z)=\mu_{z} E(z)$ where $\mu_{z}$ is the mobility of electrons in the z-direction $\left(\mu_{z} \approx 1100 \frac{\mathrm{cm}^{2}}{\mathrm{Vs}}\right.$ in CdTe). Further, the motion of a charge carrier in the sensor is described by the following equation: 


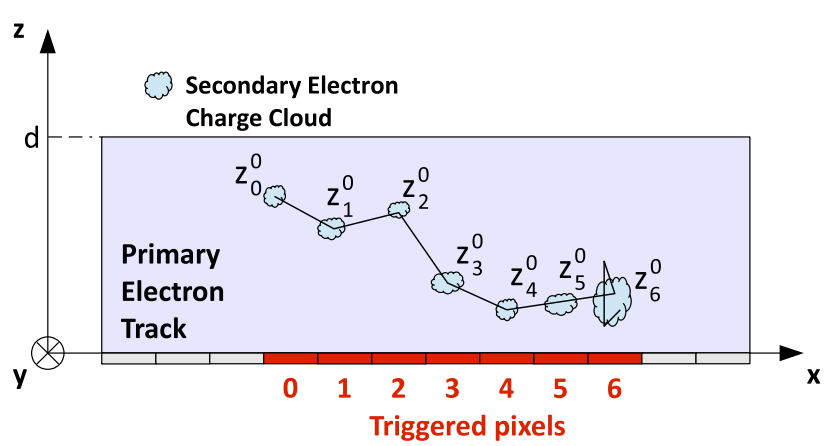

(a) $t=t_{0}$

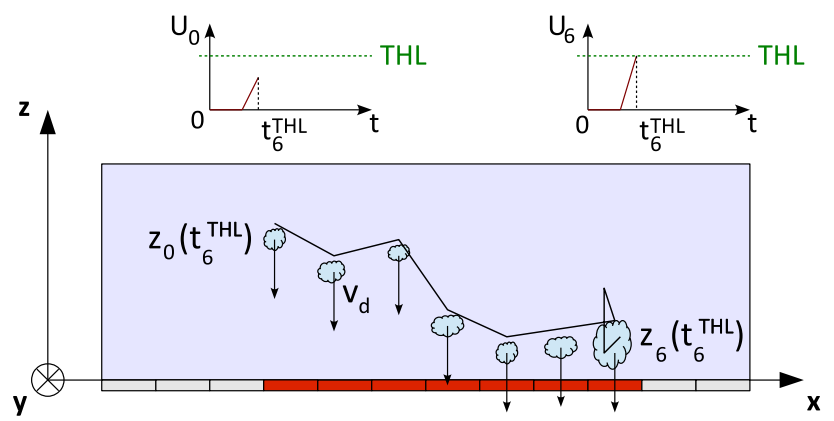

(b) $t=t_{6}^{T H L}$

Fig. 5 A cut through a pixelated detector which illustrates the goal of the reconstruction. The depth of interaction $z_{n}^{0}$ should be reconstructed for every pixel. The triggered pixels are red. a The event happens at $t_{0}$; b a snapshot at $t=t_{6}^{T H L}$ : All electron clouds were drifted towards the pixel electrodes. In pixel 6 the electron cloud is close enough to the electrode for the voltage pulse $\left(U_{6}\right)$ to exceed the threshold (THL). At the same time the voltage pulse in pixel $0\left(U_{0}\right)$ has not reached the threshold yet

$$
\begin{aligned}
z(t)= & z_{0}+\int_{0}^{t} E\left(z\left(t^{\prime}\right)\right) \mu_{z} \mathrm{~d} t^{\prime}=z_{0}+\mu_{z} U \int_{0}^{t} f_{2} \\
& +f_{1} z\left(t^{\prime}\right)+f_{3} \exp \left(-f_{4} U z\left(t^{\prime}\right)\right) \mathrm{d} t^{\prime} .
\end{aligned}
$$

Since this integral equation has no closed analytical solution for $\mathrm{z}(\mathrm{t})$, we used a numerical method to solve for $\mathrm{z}(\mathrm{t})$. We divided the total path between the anode and cathode into 20000 segments on which Eq. 2 can be approximated by a linear dependence on every segment $\mathrm{i}:|E(U, z)|=U\left(a_{i}+b_{i} \cdot z\right)$ (for every segment $i$, the parameters $a_{i}$ and $b_{i}$ are calculated). With this approximation Eq. 3 can be solved and we have a sufficient solution for $\mathrm{z}(\mathrm{t})$ on every segment.

As stated in the previous section the signal that is actually measured is the amount of charge that is induced in the pixel electrode by the charge cloud of secondary electrons during their drift through the sensor. We consider only the charge induced by the electrons and neglect the charge that is induced by the holes. The mobility of holes is $\mu_{h} \approx 100 \frac{\mathrm{cm}^{2}}{\mathrm{Vs}_{\mathrm{s}}}$, hence more than 10 times lower than the mobility of the electrons. Also the weighting potential at the cathode is less steep

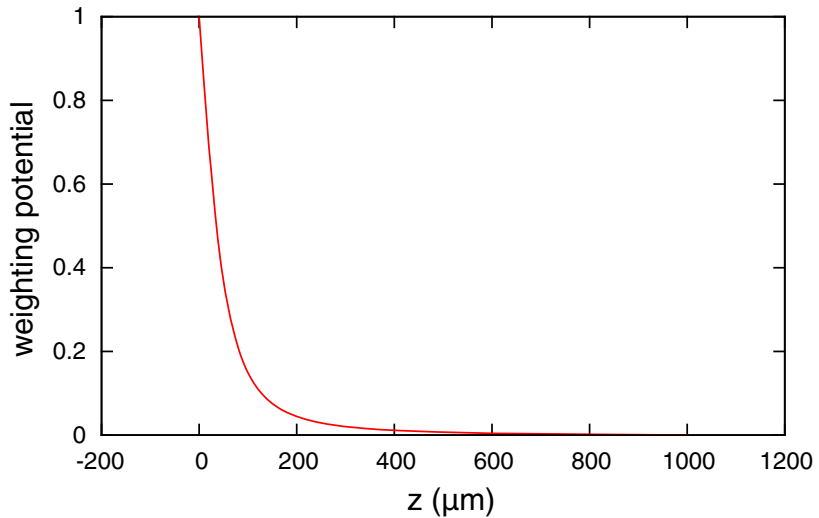

Fig. 6 The weighting potential in a $1 \mathrm{~mm}$ thick CdTe-sensor with 110 $\mu \mathrm{m}$ pixel size in $\mathrm{z}$-direction calculated with the method of Castoldi et al. [10]

than at the anode. Therefore, the main contribution of the holes to the signal happens after the integration time of the preamplifier and can be neglected.

Suppose the charge $Q_{n}^{d e p}$ was released at the time $t_{0}$ above the pixel electrode $n$ and is drifted from its initial position $z_{n}^{0}$ over a time period $t_{d}$. After this time period, the amount of charge that is induced in the pixel electrode $Q_{n}^{\text {ind }}$ can be calculated as [9]

$Q_{n}^{i n d}\left(t_{d}\right)=\left[W_{p o t}\left(z_{n}\left(t_{d}\right)\right)-W_{p o t}\left(z_{n}^{0}\right)\right] Q_{n}^{d e p}$.

The function $W_{\text {pot }}$ is called the weighting potential and takes into account the geometric properties of the sensor with its pixel electrodes. It can be determined by solving the Laplace-equation for a given geometry [10]. For our geometry of electronic pads the weighting potential along the $\mathrm{z}$-axis is shown in Fig. 6. It was calculated with the method of Castoldi et al. [10].

For every pixel the threshold equivalent charge (or energy) is known after it has been determined in a calibration process. We will call this quantity $Q_{n}^{T H L}$. Suppose this amount of charge was induced in the pixel after the time $t_{n}^{T H L}$, then Eq. 4 yields

$$
\begin{aligned}
Q_{n}^{T H L} & =Q_{n}^{\text {ind }}\left(t_{n}^{T H L}\right) \\
& =\left[W_{\text {pot }}\left(z\left(t_{n}^{T H L}\right)\right)-W_{\text {pot }}\left(z_{n}^{0}\right)\right] Q_{n}^{\text {dep }} .
\end{aligned}
$$

If a detector could measure $t_{n}^{T H L}$, the position $z_{0}^{n}$ could be determined by solving Eq. 5 . Since $z_{n}(t)$ depends also on $z_{n}^{0}$ (Eq. 3) in a non-trivial way, the equation cannot be solved analytically. Instead it can be evaluated numerically by iteration: We set $z_{0}^{n}$ to $\frac{d}{2}$ in the first $\operatorname{step}(j=1)$ and for every pixel we calculate the expression

$$
\begin{aligned}
\Delta Q_{n}\left(z_{n}^{0}\right)= & Q_{n}^{T H L}-\left[W_{p o t}\left(z_{n}\left(t_{n}^{T H L}\right)\right)-W_{p o t}\left(z_{n}^{0}\right)\right] \\
& \times Q_{n}^{\text {dep }} .
\end{aligned}
$$




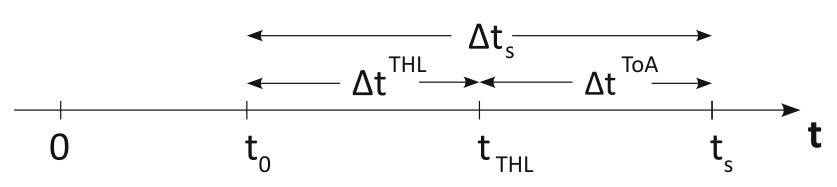

Fig. 7 An illustration of the points in time and time intervals occurring during the drift process. The interaction of the ionizing particle with the sensor happens at $t_{0}$. At $t_{T H L}$ the charge pulse (in a particular pixel) rises above the threshold. At $t_{s}$ the frame stops

In the case $\Delta Q>0$ we increase $z_{n}^{0}$ by $\Delta z_{\text {shift }}=\frac{d}{2^{j}}$ where $j$ is the step number. In the opposite case $(\Delta Q<0)$ we decrease $z_{n}^{0}$ by $\Delta z_{\text {shift }}$. The deviation of the calculated position from the actual position is smaller than $\frac{d}{2^{j}}$ after the $\mathrm{j}$-th step. Therefore, we used 30 steps in our analysis which produces a sufficient numerical accuracy for $z_{n}^{0}$.

However, an absolute measurement of $t^{T H L}$ would require to measure the time of interaction of the incident particle with the sensor. With a semiconductor this is impossible since the charge signal is always delayed by the time that it takes to induce a signal at the pixel electrode; and this time - in turn - depends on the depth of interaction.

What a detector can measure is a time-of-arrival for the charge signal in every pixel in the track relative to a fixed time-stamp set by the electronics $t_{s}$. We will call this quantity $\Delta t_{n}^{T o A}$; the time between $t_{0}$ and $t_{n}^{T H L}$ is called $\Delta t_{n}^{T H L}$; the time between $t_{0}$ and $t_{s}$ is referred to as $\Delta t_{s}$. This is illustrated in Fig. 7. According to Fig. $7 \Delta t_{n}^{T H L}$ can be calculated as

$\Delta t_{n}^{T H L}=\Delta t_{s}-\Delta t_{n}^{T o A}$.

In the last formula $\Delta t_{s}$ is unknown but this quantity is the same for every pixel. By varying $\Delta t_{s}$ the center of gravity in z-direction (the z-value averaged over all pixels) is shifted since the $\Delta t^{T o A}$ values are fixed. It can be calculated as

$\langle z\rangle=\frac{1}{Q} \sum_{n=0}^{N} z_{n}^{0} Q_{n}^{d e p}$

where $Q=\sum_{n=0}^{N} Q_{n}^{d e p}$ is the total released charge in the interaction. The ratio of the anode and cathode signal depends on the average depth of interaction [11]. It can be determined after measuring the total induced charge at the anode $Q_{a}$ $\left(Q_{c}=Q\right)$ and at the cathode $Q_{c}$ and comparing the $Q_{c} / Q_{a}$ ratio to a simulation-generated lookup-table. We will call this quantity $\langle z\rangle^{\text {meas }}$. $^{2}$

${ }^{2}$ If both sides were pixelated, the weighting potential would be flat within most of the sensor volume. Consequently $W_{p o t, A}, W_{p o t, C}$ and thus $\frac{W_{\text {pot }, A}}{W_{\text {pot }, C}}$ would be almost constant within most of the sensor volume and the determination of $\langle z\rangle^{\text {meas }}$ would not work properly. However, since the cathode is not pixelated, its weighting potential is rather linear like in a plate capacitor, which is quite different from the pixelated anode. Therefore it allows to determine $\langle z\rangle^{\text {meas }}$ from the ratio of the anode and cathode signals.
If $\Delta t_{s}$ is assumed too high, then we get $\langle z\rangle^{\text {meas }}<\langle z\rangle$ because we overestimated the drift distance. In the opposite case we get $\langle z\rangle^{\text {meas }}>\langle z\rangle$ because we underestimated the drift distance. Therefore, $\Delta t_{s}$ can be determined in the same iterative procedure as $z_{n}^{0}$ under the condition

$$
\begin{aligned}
\Delta z & =\langle z\rangle^{\text {meas }}-\langle z\rangle \\
& =\langle z\rangle^{\text {meas }}-\frac{1}{Q} \sum_{0}^{N} z_{n}\left(\Delta t_{s}\right) Q_{n}^{\text {dep }} .
\end{aligned}
$$

\subsection{Reconstruction procedure}

Based on the physics and the method described in the previous subsection a detector has to be able to measure the following quantities for a 3D track reconstruction:

- The total charge induced at the cathode $Q_{c}$.

- The charge released over every pixel $Q_{n}^{d e p}$.

- The time-of-arrival of the charge signal in every pixel $\Delta t_{n}^{T o A}$.

Based on this information, the calibration of the detector $\left(Q_{n}^{T H L}\right)$ and the properties for the sensor layer $\left(\mu, E_{z}(U, z)\right.$, $\left.W_{\text {pot }}(z)\right)$ the reconstruction algorithm operates as follows:

1. Compute numerically $z(t)$ for a given electric field configuration $E(U, z)$ in the sensor layer.

2. Determine the real average depth of interaction for the track from the $Q_{c} / Q_{a}$ ratio and the lookup-table.

3. Fix an arbitrary value for $\Delta t_{s}$ and vary $z_{n}^{0}$ for every pixel under the condition in Eq. 6 . In practice it is reasonable to choose a value between 0 and $t_{s}-t_{\max }$ for $\Delta t_{s}$ (for instance $\frac{t_{s}-t_{\max }}{2}$ ), where $t_{\max }$ is the drift time from the top of the sensor layer to the bottom.

4. Vary $\Delta t_{s}$ under the condition given by Eq. 9 and repeat the previous step to reconstruct the correct positions $z_{n}^{0}$ for every pixel.

We tested our reconstruction method on data produced by the in-house developed Monte-Carlo simulation named ROSI $[12,13]$. It is based on EGS4 and has a low energy extension with the interaction code LSCAT. For each event the simulation propagates the corresponding particle through the sensor and calculates charge deposition in the sensor layer. For a background electron of $2.8 \mathrm{MeV}^{3}$ it produces about 5000-10000 interaction points along the track. Afterwards the drift of the secondary electrons and the signal generation in the pixel electrodes is simulated. The simulation takes into account the diffusion and recombination properties of $\mathrm{CdTe}$

\footnotetext{
$32.8 \mathrm{MeV}$ is the Q-value of the double beta decay for ${ }^{116} \mathrm{Cd}$. This would be the isotope under investigation in a neutrinoless double beta experiment with Timepix-based cadmium-telluride detectors.
} 


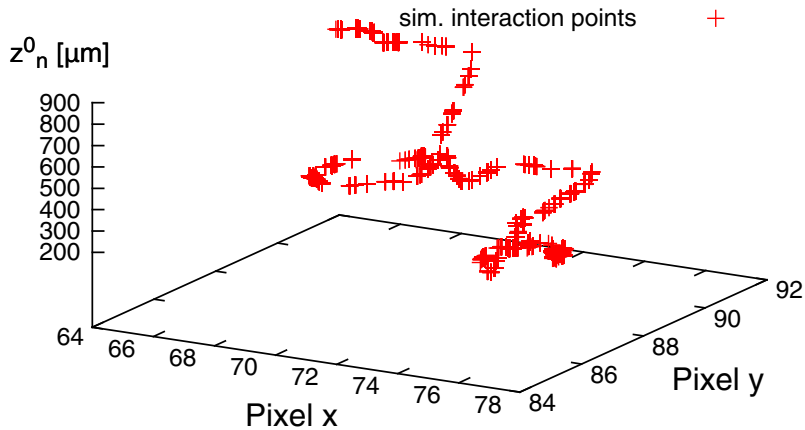

(a) Simulated Track

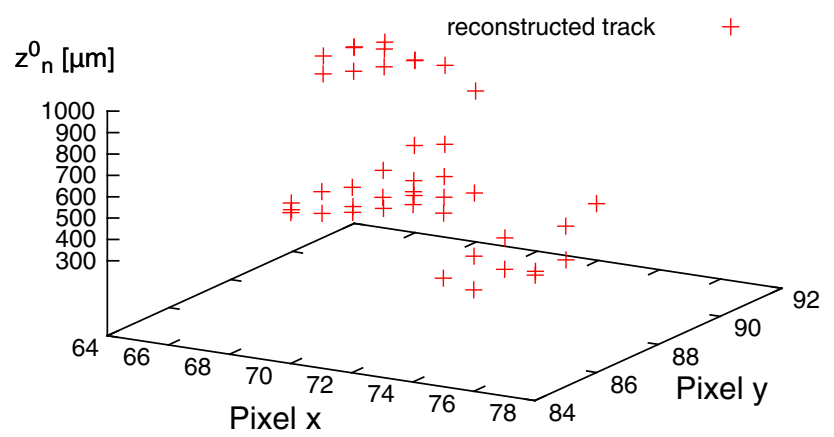

(b) Reconstructed Track

Fig. 8 Example of a simulated track (with all interaction points) and the reconstruction of this track obtained from pseudo-measured data

as well as the repulsion of the charges under their electric field.

In Fig. 8a the complete track (with all interaction points) of a $2.8 \mathrm{MeV}$ electron is shown and in Fig. $8 \mathrm{~b}$ the reconstructed track. The reconstruction uses the quantities that would be obtained with a detector of $110 \mu \mathrm{m}$ pixel size, a $1 \mathrm{~mm}$ thick sensor layer with $44.8 \mathrm{~V}$ applied bias voltage, an energy threshold of $5 \mathrm{keV}$ and a time resolution of $10 \mathrm{~ns}$ in every pixel.

The position resolution in z-direction depends on the charge resolution (which is equivalent to the energy resolution) of the cathode and anode signals as well as on the time resolution. Figure $9 \mathrm{a}$ shows the dependence of the $\mathrm{z}$ resolution $F W H M_{z}$ on the time resolution if the energy resolution $\left(\frac{\sigma(E)}{E}=2 \%\right)$ is fixed. Figure $9 \mathrm{~b}$ illustrates how the $F W H M_{z}$ depends on the energy resolution under for a fixed time resolution (10 $\mathrm{ns})$.

\section{Experimental results}

For an experimental test of our method we performed two different experiments. For both experiments we used a Timepix detector with a $1 \mathrm{~mm}$ thick cadmium-telluride sensor. The detector was bump-bonded and assembled by $\mathrm{X}$-ray Imaging

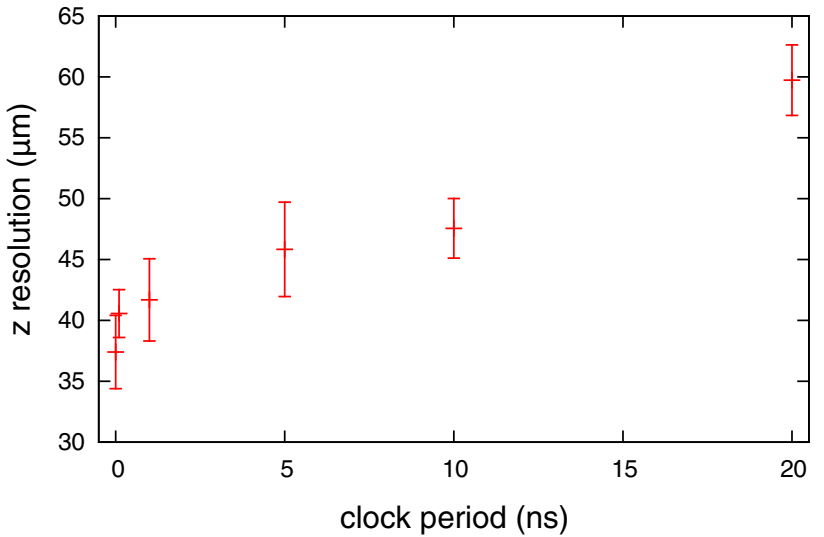

(a) Time Resolution

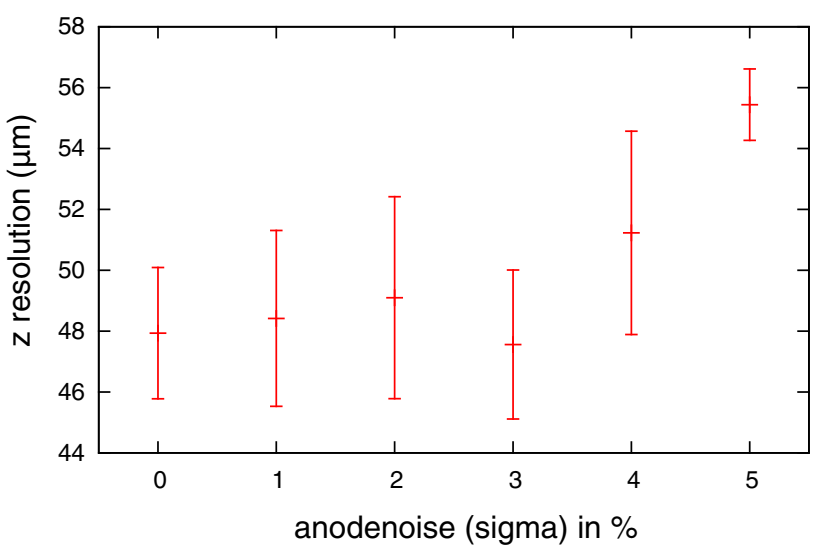

(b) Energy Resolution

Fig. 9 The interdependence between the z-position resolution $F W H M_{z}$ and the time resolution (a); and the energy resolution (b). The error bars are the given by the standard deviation if the simulation is repeated several times with the same configuration

Europe GmbH. The energy threshold of our particular detector was about $7 \mathrm{keV}$. We used the detector at a bias voltage of $44.8 \mathrm{~V}$. The reason for this is to increase the drift time of the electrons to preferably high values. In contrast to the usual application of imaging where the drift time should be as low as possible for fast counting, we need it to be rather long. The maximum clock frequency that could be used was $100 \mathrm{MHz}$ which corresponds to pulses of $10 \mathrm{~ns}$ length. At the usual voltage $(500 \mathrm{~V})$ the drift time is about $20 \mathrm{~ns}$ which is faster than the peaking time after the preamplifier (about 100 $\mathrm{ns}$ ). We need the electron drift time to be significantly larger than that in order to measure drift time differences between individual pixels. According to our simulation the drift time at $44.8 \mathrm{~V}$ is about $200 \mathrm{~ns}$. We chose a bias of about $45 \mathrm{~V}$ but not lower since the charge collection efficiency saturates at about $40-50 \mathrm{~V}$. Hence, the chosen voltage is a reasonable tradeoff between a sufficient charge collection efficiency for a good signal in each pixel and a large drift time for a "timeof-flight" position reconstruction. 


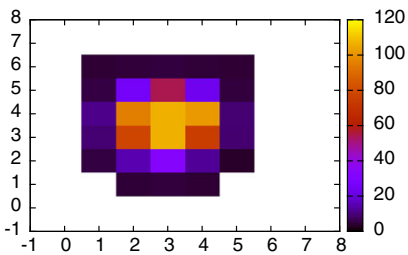

(a) $\mathrm{ToT}$

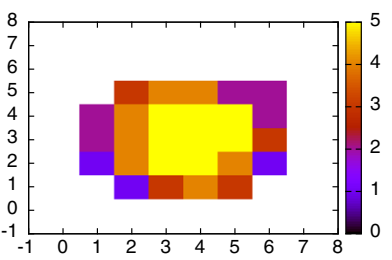

(b) $\mathrm{ToA}$

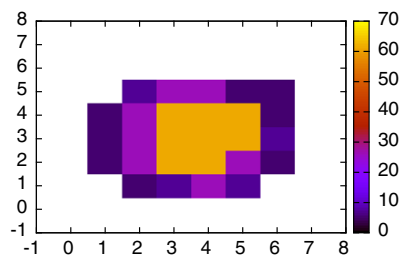

(c) Energy from ToA

Fig. 10 A typical $\alpha$-particle event measured in a ToT mode; b ToA mode. Image $\mathbf{c}$ shows the event with energy reconstructed from ToA data. The color denotes the ToT in clock cycles, the ToA in clock cycles and reconstructed energy in $\mathrm{keV}$, respectively

Before the measurements we performed a global ToT calibration for the detector. The method of calibration is described in [7]. We used two calibration energies: $59.56 \mathrm{keV}$ from ${ }^{241} \mathrm{Am}$ and $80.99 \mathrm{keV}$ from ${ }^{133} \mathrm{Ba}$.

\subsection{Reconstruction of $\alpha$-particle energy deposition distributions}

Originally, in our method the z-position (depth of interaction) for every pixel triggered in an event is reconstructed from the simultaneous measurement of the total deposited energy, the energy deposited in every pixel and a timing information in every pixel. However, according to Eq. 5 the method can also be adapted to reconstruct the energy deposition in every pixel from the timing information, the total deposited energy and the knowledge of the z-positions. In this case the quantity $Q_{n}^{i n d}$ (which is equivalent to the deposited energy) for every pixel is calculated numerically in 30 iterations with Eq. 6.

With the Timepix it is impossible to obtain all of the three quantities simultaneously. Nonetheless, in order to show the functionality of the method, it was possible to overcome this disadvantage in the following way: We placed a ${ }^{241} \mathrm{Am}$ source with an activity of about $310 \mathrm{kBq}$ close to the sensor surface. The source emits $\alpha$-particles that deposit a particular energy within the first $15 \mu \mathrm{m}$ of the sensor layer. Therefore, within good accuracy $z_{n}^{0}=0$ can be used for all pixels in the reconstruction which removes one parameter.

First, we measured the energy deposition distribution per pixel in the ToT mode. A typical event cluster in ToT mode is shown in Fig. 10a. The colour denotes the deposited energy in $\mathrm{keV}$. A cluster is a set of pixels which are direct neighbours. Usually, there are several such clusters in a single frame. An algorithm recognizes the clusters and they are treated as indi-

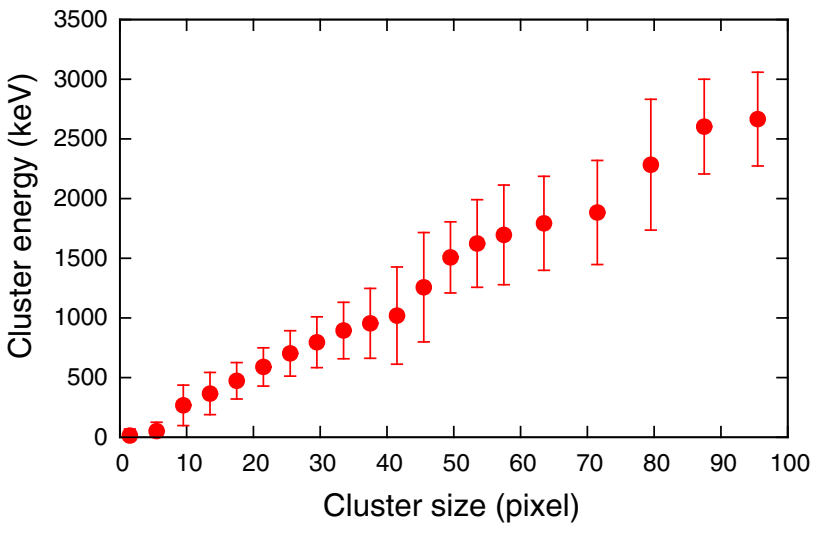

Fig. 11 The correlation between energy and cluster size from $\alpha$ particle events. The error bars show the RMS at each point

vidual events in the further analysis. By summing up all the energy values in the cluster we obtained the total deposited energy. The total deposited energy is linearly correlated with the cluster size (the number of triggered pixel in an event) as shown in Fig. 11. As the cluster size is not affected by the mode of operation, we can use this correlation to determine the total deposited energy for $\alpha$-events measured in the ToA mode.

As next, we recorded a similar number of events in the ToA and the ToT mode. A typical event in ToT, ToA and energy reconstructed from ToA are shown in Fig. 10a-c, respectively. Apparently, the dynamic range in the ToA data is lower since the total drift-time across the sensor layer is $200 \mathrm{~ns}$ and the clock that we used for this experiment was $48 \mathrm{MHz}$. Therefore, the maximum number of time slices is limited to 5 .

A comparison between the energy distribution obtained in the ToT mode and reconstructed from the data in ToA mode is shown in Fig. 12. The fraction of energy deposited in a pixel is plotted versus the distance from the center of the event. The discrepancy has multiple reasons: In the central region of the cluster the amount of released charge is so high that the induced charge goes over the threshold very quickly and the drift time differences cannot be resolved with a resolution of $21 \mathrm{~ns}$. Additionally, the charge is distributed among the pixels by charge sharing but we treat them as if the charge was initially released in the pixels. The result of this experiment suggests that the method has to be improved in the case of charge sharing but also highlights that the method works in principle.

\subsection{Reconstruction of electron tracks}

\subsubsection{Experimental design}

The second experiment was intended to demonstrate that $3 \mathrm{D}$ tracks can be reconstructed. Again, as the Timepix in its current version cannot measure all necessary quantities at the 


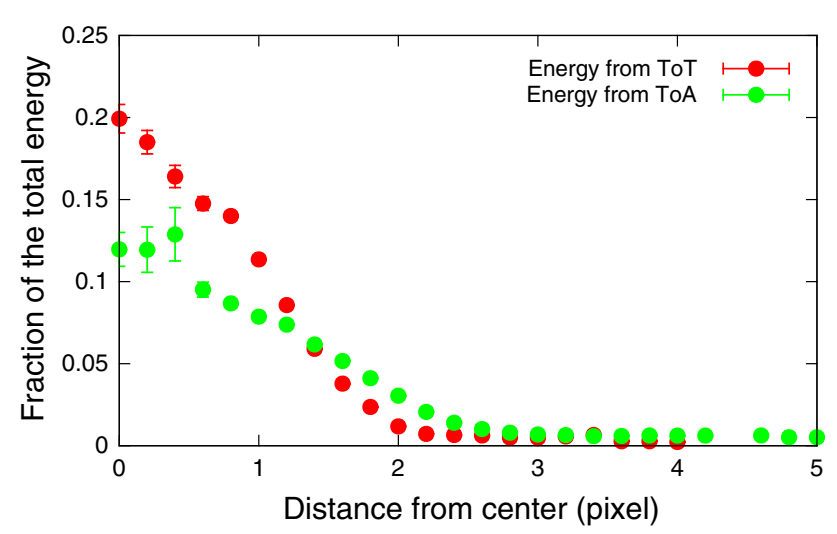

Fig. 12 The distribution of pixel energies of $\alpha$-events obtained from ToT data (red) and reconstructed from ToA data (green). The curves were averaged over many events. The error bars were calculated as the RMS divided by the square root of the number of triggered pixels in a bin. Their size is governed for example by the underlying event statistics, by the granularity of the pixel matrix and by the shape of the clusters of pixels triggered by $\alpha$-particles

same time, we designed our experiment in such a way that the parameters that cannot be measured by the detector directly, can be accessed and determined otherwise.

We used the Timepix in a so-called mixed matrix mode and took advantage of the fact that minimal ionizing particles deposit roughly the same energy in every pixel along its track. The Timepix allows to assign every pixel individually a mode of operation either ToA or ToT. For our experiment we chose the modes in a checkerboard arrangementevery pixel has a diagonal neighbour that is in the same mode and an off-diagonal neighbour that is in the different mode. It is a reasonable approximation to assume that minimal ionzing particles deposit nearly the same energy in every pixel. Hence, one can determine the energy deposited in a pixel that runs in the ToA mode by calculating the average value of deposited energy in the surrounding triggered ToT pixels. The second advantage of minimal ionizing particles is that they propagate through the complete sensor on an almost straight line. Thus, we know that the average z-position of the track is $\langle z\rangle^{\text {meas }}=\frac{d}{2}$ without measuring the total charge at the common electrode.

Another way to determine the averaged deposited energy in every pixel is to take data in ToT mode and afterwards without changing the angle between the beam and the sensor layer to take data in ToA mode. From the ToT measurement we can determine the energy deposition in every pixel and afterwards the ToA data can be used for z-position reconstruction under the assumption that approximately the same energy was deposited in every pixel for each measurement in ToT and ToA mode under a fixed angle.

The data with minimal ionizing particles was acquired at the DESY T21 testbeam. We used an electron beam at 4.4 $\mathrm{GeV}$. The sensor layer was positioned under two different angles to the beam direction. In one case the angle was about

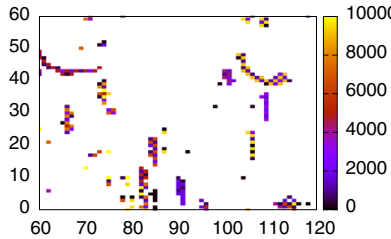

(a) Mixed Mode

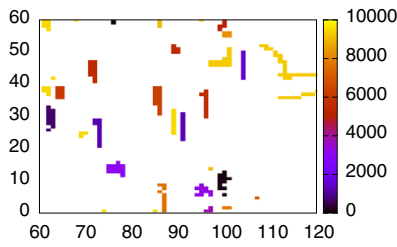

(c) ToA Mode

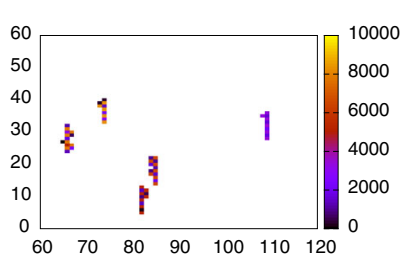

(b) Mixed Mode
Fig. 13 Typical tracks for the evaluation of the z-resolution before (a, c) and after $(\mathbf{b}, \mathbf{d})$ track selection in the mixed-mode $(\mathbf{a}, \mathbf{b})$ and in the ToA mode (c, d). The colour bar denotes the measured ToA or ToT in every pixel

$42^{\circ}$ and we obtained short tracks of 8-9 pixels on average and in the other case $\left(10^{\circ}\right)$ the tracks were 55-56 pixels long in average. The frame-time was $120 \mu$ s wherefore two tracks never overlap on one frame. We took data in the mixed, the ToT and the ToA mode.

\subsubsection{Data analysis and results}

For the evaluation of the resolution that we achieved in $\mathrm{z}-$ direction we performed a selection of tracks and sorted out tracks which are not meaningful due to the following criteria: first, we rejected tracks where at least one pixel counted a ToA value of at least 9465 which is the maximal ToA value that can be expected with the used frame time and clock frequency. With this cut, tracks were ignored which occurred shortly before or right at the beginning of the frame. For these tracks the exact moments of detection cannot be determined due to the ToA measurement principle of the Timepix. Secondly, the electron beam was in x-direction while the detector was placed in the yz-plane and then tilted along the $y$-axis. Thus, as the tracks from minimal ionizing events are straight lines, meaningful events should trigger pixels mostly in one x-column. For events which do not produce straight lines due to scattering in the sensor our reconstruction will give false results since the assumption $\langle z\rangle^{\text {meas }}=\frac{d}{2}$ is not fulfilled. Hence, we used the following additional criterion for event selection: Only tracks which had twice as much triggered pixels in one x-column than in other columns combined are taken into account. Since hardly any long tracks fulfilled this criteria, we used only the short tracks for the further analysis. At last, we used only tracks with at least 10 triggered pixels to avoid background from low energy events. Figure 13a-d show a sample of tracks before and after event 


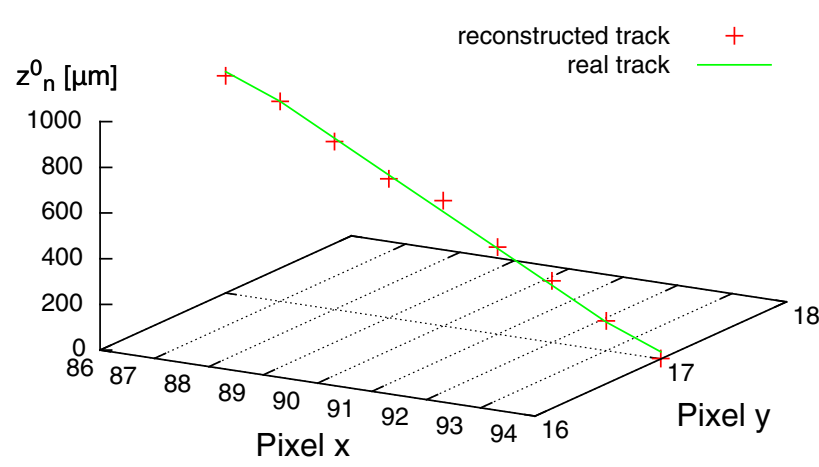

(a) Short Track

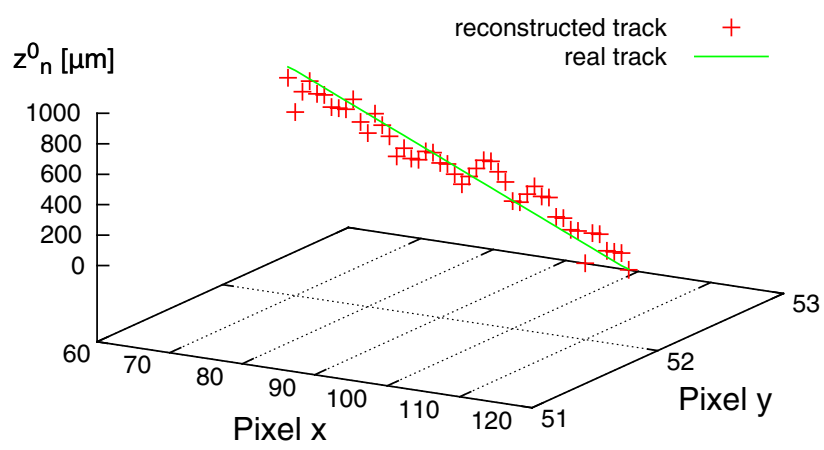

(b) Long Track

Fig. 14 A reconstructed track of a typical short and long track after reconstruction in comparison to the real track. The tracks go parallel to the $\mathrm{x}$-axis wherefore the $\mathrm{y}$-values are the same for all voxels

selection in the mixed-mode and in the ToA mode, respectively.

The experiment with $\alpha$-particles showed that our algorithm can give unsatisfying results for charge sharing pixels. Therefore, we removed pixels which are not in the main $\mathrm{x}$ column as these are probably due to charge sharing. Also, we removed pixels with the highest y-coordinate values as these are most probably due to charge sharing from the pixels where the electron entered and left the sensor layer.

A typical track in comparison to the expected track for each case (long and short) is shown in Fig. 14a, b, respectively. At this point it is important to mention that the algorithm is not biased in any way to reconstruct a straight line. The fact that the result of reconstruction is a straight line indeed is a strong indicator that our method works properly. The reconstructed points are scattered close around the actual particle trajectory which indicates a meaningful result.

We calculated the z-resolution as the root mean square (RMS), where the reference $\mathrm{z}$-value in every point was determined by a line fitted through the pixels with the highest and the lowest y-values (green line in Fig. 14). This line is what comes closest to the real particle trajectory. The results are

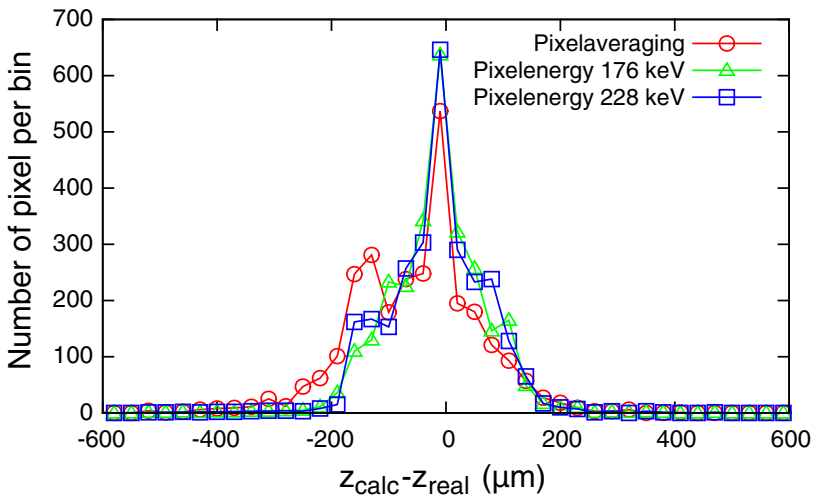

(a) Mixed Mode

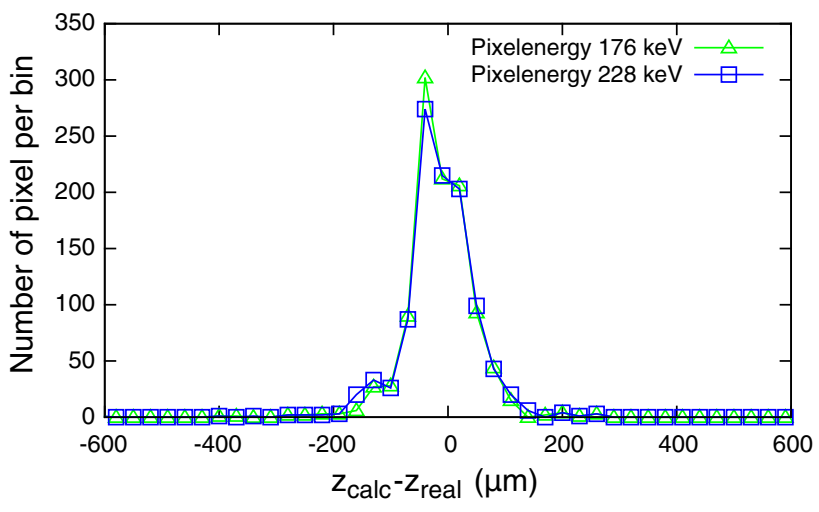

(b) ToA Mode

Fig. 15 The deviations of the reconstructed z-position from the actual track for events measured in mixed mode (a) and ToA mode (b)

Table 1 Results on the z-resolution obtained in different modes of operation

\begin{tabular}{lcl}
\hline & Mixed mode $(\mu \mathrm{m})$ & ToA mode \\
\hline Neighbours averaging & 119 & - \\
All pixel at $228 \mathrm{keV}$ & 91 & $66 \mu \mathrm{m}$ \\
All pixel at $176 \mathrm{keV}$ & 89 & $63 \mu \mathrm{m}$ \\
\hline
\end{tabular}

summarized in Table 1. Figure 15a, b shows the deviations of the reconstructed z-position from the actual track.

In the mixed-mode we achieved a z-resolution of 119 $\mu \mathrm{m}$. By using the data from ToA only and a fixed energy of $228 \mathrm{keV}$ we achieve a z-resolution of $66 \mu \mathrm{m}$. This value for the average energy was determined from the ToT measurement as the average deposited energy per pixel. However, since this energy is a constant for all pixels in the reconstruction, it can also be set to a different fixed value. It turns out that the best resolution of $63 \mu \mathrm{m}$ could be achieved by using the data from ToA mode and a fixed energy of $176 \mathrm{keV}$ for every pixel. For the mixed-mode we obtained a resolution of $91 \mu \mathrm{m}$ with a fixed energy of $228 \mathrm{keV}$ and $89 \mu \mathrm{m}$ with a fixed energy of $176 \mathrm{keV}$. 
The experiments were limited to straight tracks from minimal ionizing particles since the available detectors cannot provide all the information required for reconstruction. Nonetheless the next generation of Timepix detectors (Timepix3) [14] will provide a simultaneous read-out of timing and energy deposition information per pixel. Also the readout of a backside signal could be included as for a coplanar-grid-type of detector. With a proposed time resolution of $1.6 \mathrm{~ns}$, a voxel detector with about $50 \mu \mathrm{m}$ voxel size can be expected. A Timepix3 detector with a CdTe sensor could therefore provide an interesting opportunity for new imaging techniques with voxel detectors.

\section{Conclusions and outlook}

In this publication we presented and evaluated a method to reconstruct $3 \mathrm{D}$ particle trajectories through a sensor layer of a pixelated semiconductor detector. Our simulations suggests that a z-resolution of about 40-50 $\mu \mathrm{m}$ can be achieved under realistic detector performance assumptions.

As a major result, we successfully demonstrated that the method works experimentally and a particle track can be reconstructed from actual data. The achieved z-resolution was $63 \mu \mathrm{m}$ in the best case.
Acknowledgments We would like to thank Dominik Dannheim and Ralf Diener for their help and giving us the opportunity to acquire data at the DESY test beam facility. Also, we would like to thank the Medpix Collaboration for their support. We are grateful to the Deutsche Forschungsgemeinschaft for supporting Thomas Gleixner.

Open Access This article is distributed under the terms of the Creative Commons Attribution License which permits any use, distribution, and reproduction in any medium, provided the original author(s) and the source are credited.

Funded by SCOAP ${ }^{3}$ / License Version CC BY 4.0.

\section{References}

1. J. Schechter, J. Valle, Phys. Rev. D 25, 2951 (1982)

2. P. Vogl, S. Elliott, Ann. Rev. Nucl. Part. Sci. 52, 115 (2002)

3. B. Plimley et al., Nucl. Instrum. Methods A 654, 244 (2011)

4. J. Kim et al., Nucl. Instrum. Methods A 683, 53 (2012)

5. X. Llopart et al., Nucl. Instrum. Methods A 581, 485 (2007)

6. V. Kraus et al., JINST 6, C01079 (2011)

7. M. Filipenko et al., Eur. Phys. J. C 73, 2374 (2013)

8. T. Michel et al., Adv. High Energy Phys. 2013, 105318 (2013)

9. W. Shockley, J. Appl. Phys. 9, 635 (1938)

10. A. Castoldi et al., IEEE Trans. Nucl. Sci. 43(1), 256 (1996)

11. W. Li et al., IEEE Trans. Nucl. Sci. 47(3), 890 (2000)

12. J. Giersch et al., Nucl. Instrum. Methods A 509, 151 (2003)

13. J. Durst, J. Giersch, Nucl. Instrum. Methods A 591, 300 (2007)

14. M. van Beuzekom et al., in Proceedings of Science (SISSA, 2011), pp. 1-8 